\title{
NONLINEAR REPETITIVE CONTROL OF THE METAL CUTTING MACHINE FEED MODULE WITH SATURATED INPUT
}

\author{
Eugenie L. Eremin \\ Information and Control Systems Department \\ Amur State University \\ Russia \\ ereminel@mail.ru
}

\author{
Larisa V. Nikiforova \\ Information and Control Systems Department \\ Amur State University \\ Russia \\ chepak@inbox.ru
}

\author{
Evgeniy A. Shelenok \\ Automation and System Engineering Department \\ Pacific National University \\ Russia \\ cidshell@mail.ru
}

Article history:

Received 15.11.2021, Accepted 20.12.2021

\begin{abstract}
The article discusses the methodology for constructing an automatic control system for the feed module of the cutting tool for lathes in the presence of input saturation. The presence of the saturation function is due to restrict unwanted movements of the feed module actuator. The generator for periodic signals, the hyperstability criterion, $L$-dissipativity conditions and an implicit reference model are used as the solution methods for structural synthesis of the control system. At the stage of simulation, the functioning quality of the developed control system is illustrated.
\end{abstract}

\section{Key words}

Combined control algorithm, generator for periodic signals, hyperstability criterion, $L$-dissipativity, servo system, repetitive control system.

\section{Introduction}

One of the most important problems of the modern theory and practice of automatic control is the problem of developing regulators for tool feed electric drives of the metal-cutting machines for processing non-circular parts ([Nikolsky, 2012], [Nikolsky, 2016], [Li, Li , 1992], [Yurkevich, 2001]). Such systems are characterized by the cyclical nature of the reference action, which determines the desired movement of the cutter. Also, these systems are subject to the influence of repeating external disturbances arising due to elastic and thermal deforma- tions during the processing of metal workpieces. In this case, one of the most expedient methods for the synthesis of such systems is so-called method of repetitive control. The distinctive feature of this method is the presence in the control system main loop of the generator for periodic signals which is capable to reproduce any cyclical signals in order to track and/or parry them ([Hara et al., 1988], [Yao et al., 2013], [Ishii, Yamamoto, 1998], [Jiang et al., 1995], [Ramos et al., 2020], [Nikolova, 2019], [Toujeni et al., 2020], [Alsubaie et al., 2018], [Ma et al., 2020], [Ghosh, Paden, 2000]). This is due to the fact that it is necessary to obtain a solution that, on the one hand, will provide high-precision processing of the periodic reference signal (the required movement of the machine tool). On the other hand it will compensate the external force disturbance, which is also cyclical.

As it's shown in [Eremin, Shelenok, 2018(2)], [Eremin, Shelenok, 2018] and [Eremin, Shelenok, 2018(1)] the development of repetitive control systems can be carried out with the help of hyperstability criterion and concept of $L$-dissipativity. In this paper, we propose the nonlinear regulator for repetitive control system of mechatronic module for cutting tool in the presence of input saturation.

\section{Preliminaries}

We use the data of the acceleration curves of the electric drive of the lathes feed (Fig. 1) to construct the mathematical model of the considered control plant. On Fig. 1 a set of points represents the transient process of real 
control plant, solid lines show the boundaries of the region of its approximation, formed by fourth order inertia links

$$
W(s)=\frac{K}{s^{4}+a_{4} s^{3}+a_{3} s^{2}+a_{2} s+a_{1}},
$$

where $K$ and $a_{i}, i=1, \ldots, 4$ are unknown coefficients determined with precision to ranges

$$
\begin{gathered}
K^{-}=18.6 \leq K \leq 19.5=K^{+}, \\
a_{1}^{-}=18.4 \leq a_{1} \leq 23.5=a_{1}^{+}, \\
a_{2}{ }^{-}=41,4 \leq a_{2} \leq 64=a_{1}^{+}, \\
a_{3}{ }^{-}=45.7 \leq a_{3} \leq 50=a_{3}^{+} \\
a_{4}{ }^{-}=8.5 \leq a_{4} \leq 16.5=a_{4}^{+} .
\end{gathered}
$$

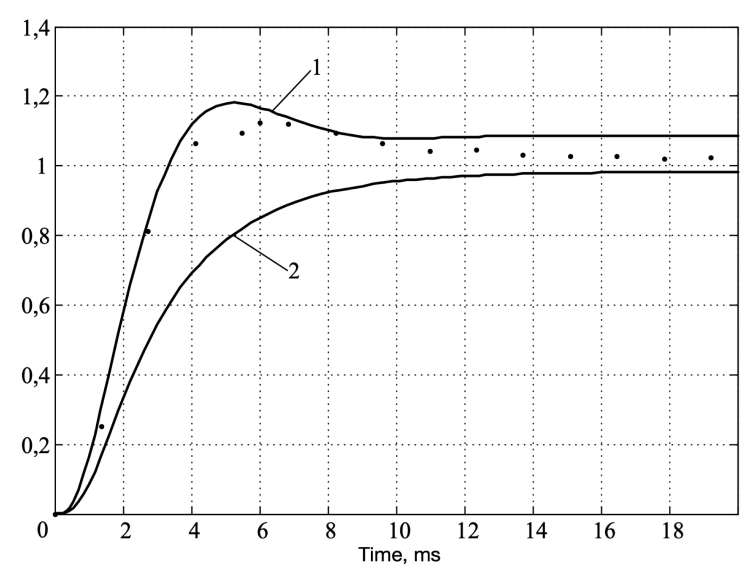

Figure 1. Accelerating characteristic of the electric drive

On Fig. 1: curve 1 corresponds to maximum values of $a_{i}, K$. Curve 2 corresponds to their minimum values.

Taking into account that the plant (1) is a subject to external disturbances and requires to limit the input control signal in order to prevent unwanted displacements of the cutting tool, we represent the mathematical model (1) in the following input-state-output form:

$$
\begin{gathered}
\frac{d \mathbf{x}(t)}{d t}=\mathbf{A} \mathbf{x}(t)+\mathbf{b}[S(u(t))+f(t)], \\
y(t)=\mathbf{L}^{T} \mathbf{x}(t)=x_{1}(t), \\
\mathbf{A}=\left(\begin{array}{cccc}
0 & 1 & 0 & 0 \\
0 & 0 & 1 & 0 \\
0 & 0 & 0 & 1 \\
a_{1} & a_{2} & a_{3} & a_{4}
\end{array}\right), \mathbf{b}=\left(\begin{array}{c}
0 \\
0 \\
0 \\
K
\end{array}\right), \mathbf{L}=\left(\begin{array}{l}
1 \\
0 \\
0 \\
0
\end{array}\right) .
\end{gathered}
$$

where $\mathbf{x}(t) \in R^{4}$ is the state variables vector; $\mathbf{A}, \mathbf{b}$ are state matrix and control vector respectively with uncer- tain parameters; $u(t) \in R$ and $y(t) \in R$ are control signal and output of the plant; $\mathbf{L}$ is the output vector; $f(t)$ are external bounded permanent disturbances; $S(u(t))$ is the nonlinear saturation function (Fig. 2) which has the form

$$
S(u(t))= \begin{cases}S_{0}, & u(t)>S_{0} \\ u(t), & |u(t)| \leq S_{0} \\ -S_{0}, & u(t)<-S_{0}\end{cases}
$$

$S_{0}>0$ is a known constant corresponding to the limitation level.

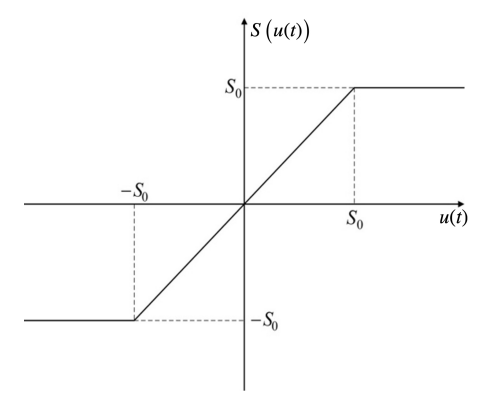

Figure 2. Graph of the saturation function

The obtained plant's mathematical model (2) - (4) has an equivalent analog in the input-output form:

$$
y(t)=W(p)\{S(u(t))+f(t)\}
$$

where $p=d / d t ; y(t)$ is the output signal; $W(p)$ is the transfer function.

We suppose that the plant (5) operates under the following assumptions:

1. External force disturbances satisfy the inequality:

$$
|f(t)| \leq f_{0}, f_{0}=\text { const }>0, \forall t \neq 0,
$$

where $f_{0}$ is a known constant.

2. Parameters of $f(t)$ are priori unknown: $f(t)=$ $=f_{\xi}(t), \xi$ are unknown parameters which incudes in the known bound numerical set $\Xi$.

3. The transfer function of the plant (5) has the following form: $W(p)=\frac{K}{a(p)}, a(p)=p^{4}+a_{4} p^{3}+$ $+a_{3} p^{2}+a_{2} p+a_{1}$, where $a(p)$ is the normalized polynomial with unknown coefficients and an arbitrary roots.

4. For direct measurement only the output signal $y(t)$ is available. 
Let us pass the signal $y(t)$ through the output correction filter $(O C F)$ [Eremin et al., 2020]

$$
y_{F}(t)=W_{F}(p) y(t)=\left(\frac{T p+1}{T^{*} p+1}\right)^{3} y(t),
$$

where $y_{F}(t)$ and $y(t)$ are filter output and input signals respectively; $W_{F}(p)$ is the filter transfer function; $T$ and $T^{*}$ are correcton filter time constants; the value of $T^{*}$ is small.

In this case the model of the serial connection of the plant (5) and the $O C F$ (6) can be represented in the following form

$$
\begin{gathered}
y_{F}(t)=W_{F}(p) \cdot W(p)\{S(u(t))+f(t)\}= \\
=\left(\frac{T p+1}{T^{*} p+1}\right)^{3} \cdot \frac{K}{a(p)}\{S(u(t))+f(t)\}= \\
=\frac{\tilde{b}(p)}{\tilde{a}(p)}\{S(u(t))+f(t)\}= \\
=\tilde{W}(p)\{S(u(t))+f(t)\} \\
\tilde{a}(p)=a(p)\left(T^{*} p+1\right)^{3}, \tilde{b}(p)=K(T p+1)^{3} .
\end{gathered}
$$

The real connection of the $O C F$ and the control plant (7) can always be associated with their "virtual" serial connection:

$$
\begin{gathered}
\tilde{W}(p)=\frac{K}{a(p)} \cdot\left(\frac{T p+1}{T^{*} p+1}\right)^{3}= \\
=\frac{\tilde{b}(p)}{a(p)} \cdot \frac{1}{\left(T^{*} p+1\right)^{3}}=\hat{W}(p) \cdot \frac{1}{\left(T^{*} p+1\right)^{3}} .
\end{gathered}
$$

As it's shown in [Eremin et al., 2020] due to a small time constant $T^{*}$ we can write the fair relation $\frac{1}{\left(T^{*} p+1\right)^{3}} \cong 1$ and replace model (7) with the approximate mathematical model

$$
\begin{gathered}
y_{F}(t) \cong \frac{\tilde{b}(p)}{a(p)} y(t)=\hat{W}(p)\{S(u(t))+f(t)\}, \\
\tilde{b}(p)=K(T p+1)^{3}=\tilde{b}_{3} p^{3}+\tilde{b}_{2} p^{2}+\tilde{b}_{1} p+\tilde{b}_{0},
\end{gathered}
$$

or in the state-space like

$$
\begin{gathered}
\frac{d \mathbf{x}(t)}{d t}=\mathbf{N} \mathbf{x}(t)+\mathbf{b}\left\{\mathbf{a}^{T} \mathbf{x}(t)+\right. \\
+S(u(t))+f(t)\}, y_{F}(t)=\mathbf{c}^{T} \mathbf{x}(t), \\
\mathbf{x}\left(t_{0}\right)=\mathbf{x}_{0}, t \geq t_{0}=0,
\end{gathered}
$$

where $\mathbf{x}(t)=\left[x_{1}(t), x_{2}(t), x_{3}(t), x_{4}(t)\right]^{T}$ is the state variables vector; $N$ is the nilpotent matrix of $(4 \times 4)$ size; $\mathbf{b}=[0,0,0,1]^{T}$ is stationary vector; $\mathbf{a}^{T}=$ $=\left[a_{1}, a_{2}, a_{3}, a_{4}\right]$ and $\mathbf{c}^{T}=\left[\tilde{b}_{0}, \tilde{b}_{1}, \tilde{b}_{2}, \tilde{b}_{3}\right]$ are vectors with the appropriate coefficients.

\section{Control Goals and Reference Model}

Let us formulate two control goals for the considered system (1) - (10).

\subsection{Main Control Goal}

It is required to provide a high-precision tracking of the plant's (5) output signal to the command signal $r(t)$, which sets the required processing profile of the product (the required movement of the cutting tool):

$$
\begin{gathered}
\lim _{t \rightarrow \infty}|r(t)-y(t)| \leq \Delta_{r}, \\
\Delta_{r}=\text { const }>0,
\end{gathered}
$$

where $\Delta_{r}$ is a small value relatively to the maximum value of the command signal $r(t)$ (required tracking accuracy).

\subsection{Additional Control Goal}

If the required dynamics of the system main output $y_{F}(t)$ is determined, by analogy with [Eremin et al., 2020], using the command correction filter $(C C F)$ which describes like

$$
\hat{r}(t)=W_{F}(p) r(t)=\left(\frac{T p+1}{T^{*} p+1}\right)^{3} r(t),
$$

where $\hat{r}(t)$ is the auxiliary command signal; then an additional control goal can be formulated as following: it is required to synthesize an explicit form of the control law $u(t)=u\left(y_{F}(t), \hat{r}(t)\right)$ that ensures the fulfillment of the following objective

$$
\begin{gathered}
\lim _{t \rightarrow \infty}\left|\hat{r}(t)-y_{F}(t)\right| \cong\left|y^{*}(t)-y_{F}(t)\right| \leq \hat{\Delta}_{r}, \\
\hat{\Delta}_{r}=\text { const }>0
\end{gathered}
$$

where $\hat{\Delta}_{r}$ is a small constant; $y^{*}(t)$ is the output of $\mathrm{im}$ plicit reference model (IRM):

$$
\begin{gathered}
y^{*}(t)=\frac{1}{\chi_{*}^{-1} p+1} \hat{r}(t)=\frac{\chi_{*}}{p+\chi_{*}} \hat{r}(t), \\
\chi_{*}=\text { const }>0,
\end{gathered}
$$

which at $\chi_{*}>>0$ can be represented as $y^{*}(t) \cong \hat{r}(t)$. Instead of reference (14) we will use its equivalent ana$\log$ to synthesize the control law:

$$
\begin{gathered}
y^{*}(t)=\frac{\chi_{*} \tilde{b}(p) \tilde{b}_{3}}{\left(p+\chi_{*}\right) \tilde{b}(p) \tilde{b}_{3}} \hat{r}(t)= \\
=\frac{\hat{\chi_{*}} \tilde{b}(p) \tilde{b}_{3}^{-1}}{\left(p+\chi_{*}\right) \tilde{b}(p)} r(t), \hat{\chi}_{*}=\chi_{*} \tilde{b}_{3} .
\end{gathered}
$$


Mathematical model (15) in the state-space can be represented in the following form:

$$
\begin{gathered}
\frac{d \mathbf{x}^{*}(t)}{d t}=\mathbf{A}^{*} \mathbf{x}^{*}(t)+\mathbf{b} \hat{\chi_{*}} \hat{r}(t), \\
y^{*}(t)=\mathbf{c}^{T} \mathbf{x}^{*}(t), \mathbf{x}\left(t_{0}\right)=0, t \geq t_{0}=0,
\end{gathered}
$$

where $\mathbf{x}^{*}(t)=\left[x_{1}^{*}(t), x_{2}^{*}(t), x_{3}^{*}(t), x_{4}^{*}(t)\right]^{T}$ is the reference state vector; $\mathbf{A}^{*}=\mathbf{N}+\mathbf{b a}_{*}^{T}=\mathbf{N}+$ $\mathbf{b}\left(\mathbf{a}-\chi_{*} \mathbf{c}\right)^{T}$ is the Hurwitz matrix of $(4 \times 4)$ size; $\mathbf{a}_{*}^{T}=\left(\mathbf{a}-\chi_{*} \mathbf{c}\right)^{T}=\left[a_{* 1}, a_{* 2}, a_{* 3}, a_{* 4}\right]=$ $=\left[a_{1}-\chi_{*} \tilde{b}_{3}, a_{2}-\chi_{*} \tilde{b}_{3}, a_{3}-\chi_{*} \tilde{b}_{3}, a_{4}-\chi_{*} \tilde{b}_{3},\right]$ is vector with given coefficients.

\section{Main Results}

We will use the hyperstability criterion for designing the nonlinear repetitive control law.

Considering the vector of deviations $\mathbf{e}(t)=\mathbf{x}^{*}(t)-$ $-\mathbf{x}(t)$, we can write the equivalent mathematical description of the system in the following form

$$
\begin{gathered}
\frac{d \mathbf{e}(t)}{d t}=\mathbf{A}^{*} \mathbf{e}(t)+\mathbf{b} \mu(t), v(t)=\mathbf{c}^{T} \mathbf{e}(t)= \\
=\hat{r}(t)-y_{F}(t), \\
\mu(t)=\theta(t)-\chi_{*} y_{F}(t)-u(t)- \\
-[S(u(t))-u(t)],
\end{gathered}
$$

where $v(t)$ and $\mu(t)$ are transformed output and control signals respectively; $\theta(t)=\theta(t+T)=\chi_{*}-f(t)$ is the periodic signal.

In accordance with the requirements of the hyperstability criterion ([Eremin, Shelenok, 2018(2)], [Eremin, Shelenok, 2018], [Eremin, Shelenok, 2018(1)], [Eremin et al., 2020]) for the equivalent system (13), it is necessary to fulfill two conditions:

$$
\begin{gathered}
\operatorname{Re}\left[\mathbf{c}^{T}\left(j \omega \mathbf{E}-A^{*}\right)^{-1} \mathbf{b}\right]>0, \forall \omega \geq 0, \\
\eta(0, t)=-\int_{0}^{t} \mu(\varsigma) v(\varsigma) d \varsigma \geq \eta_{0}^{2}, \\
\eta_{0}=\text { const }>0, \forall t>0 .
\end{gathered}
$$

The validity of the frequency condition (18) is obvious, since the transfer function of the system (17) linear part coincides with the description of the first order inertial link:

$$
W(p)=\mathbf{c}^{T}\left(p \mathbf{E}-A^{*}\right)^{-1} \mathbf{b}=\frac{\chi_{*}}{p+\chi_{*}} .
$$

Let us ensure the fulfillment of the second condition (19). We represent the control signal as $u(t)=u_{1}(t)+$ $u_{2}(t)$ and transform the left side of the integral inequality (19) like

$$
\begin{gathered}
\eta(0, t)=\int_{0}^{t}\left(u_{1}(\varsigma)-\theta(\varsigma)\right) v(\varsigma) d \varsigma+ \\
+\left(\int_{0}^{t} u_{2}(\varsigma) v(\varsigma) d \varsigma+\int_{0}^{t} \chi_{*} y_{F}(\varsigma) v(\varsigma) d \varsigma\right)+ \\
+\int_{0}^{t}[S(u(\varsigma))-u(\varsigma)] v(\varsigma) d \varsigma= \\
=\sum_{i=1}^{2} \eta_{i}(0, t)+\int_{0}^{t}[S(u(\varsigma))-u(\varsigma)] v(\varsigma) d \varsigma .
\end{gathered}
$$

We syntesize $u_{2}(t)$ in the form of generator for periodic signals:

$$
\begin{gathered}
u_{1}(t)=u_{1}(t-T)+\gamma_{1} v(t) \tilde{\vartheta}(t), \\
u_{1}(h)=0, h \in[-T ; 0],
\end{gathered}
$$

where $\gamma_{1}=$ const $>0 ; T=$ const $>0 ; \tilde{\vartheta}(t)$ is ouput signal of the dynamic switch

$$
\begin{gathered}
\tilde{\tau} \frac{d \tilde{\vartheta}(t)}{d t}+\tilde{\vartheta}(t)=\vartheta(t), \tilde{\vartheta}(0)=0, \\
\vartheta(t)= \begin{cases}1, & \forall[S(u(t))-u(t)] v(t) \geq 0, \\
\vartheta_{0}, & \forall[S(u(t))-u(t)] v(t)<0,\end{cases}
\end{gathered}
$$

$\tilde{\tau}$ is the switch time constant; $\vartheta(t)$ is the switching function; $0<\vartheta_{0}<1$ is the scaling coefficient.

Then for $\eta_{1}(0, t)$ we can obtain following fair estimate (see [Eremin, Shelenok, 2018(2)] and [Eremin, Shelenok, 2018(1)]:

$$
\begin{gathered}
\eta_{1}(0, t) \geq \gamma_{1} \vartheta_{0} \int_{0}^{t} v(\varsigma) \times \\
\times\left[\int_{0}^{\varsigma} \omega_{0}(\varsigma-\rho) v(\rho) d \rho-\theta(\varsigma)\right] d \varsigma \geq-\eta_{01}^{2}, \\
\eta_{01}^{2}=\text { const }, \forall t>0
\end{gathered}
$$

where $\omega_{0}(\cdot)$ is the weight function of the generator for periodic signals (periodic integrator). Its transfer function has the form $W(s)=\frac{\beta}{1-\exp (-s T)}, \beta=$ $=$ const $\geq 1$.

If we synthesize $u_{2}(t)$ in the following combined form

$$
\begin{aligned}
u_{2}(t)= & \gamma_{21} y_{F}(t) \int_{0}^{t} y_{F}(\varsigma) v(\varsigma) \tilde{\vartheta}(t)+ \\
& +\gamma_{22} y_{F}^{2}(t) v(t) \tilde{\vartheta}(t), \\
\gamma_{21}= & \text { const }>0, \gamma_{22}=\text { const }>0
\end{aligned}
$$


then for summand $\eta_{2}(0, t)$ from (21) we can obtain the following integral estimate:

$$
\begin{gathered}
\eta_{2}(0, t)=\gamma_{21} \int_{0}^{t} y_{F}(\varsigma) v(\varsigma) \times \\
\times \int_{0}^{\varsigma} y_{F}(\rho) v(\rho) \tilde{\vartheta}(\rho) d \rho d \varsigma+ \\
+\gamma_{22} \int_{0}^{t} y_{F}^{2}(\varsigma) v^{2}(\varsigma) \tilde{\vartheta}(\varsigma) d \varsigma+ \\
+\chi_{*} \int_{0}^{t} y_{F}(\varsigma) v(\varsigma) d \varsigma \geq \gamma_{21} \vartheta_{0} \int_{0}^{t} y_{F}(\varsigma) v(\varsigma) \times \\
\times \int_{0}^{\varsigma} y_{F}(\rho) v(\rho) d \rho d \varsigma+\gamma_{22} \vartheta_{0} \int_{0}^{t}\left(y_{F}(\varsigma) v(\varsigma)\right)^{2} d \varsigma+ \\
+\chi_{*} \int_{0}^{t} y_{F}(\varsigma) v(\varsigma) d \varsigma \geq \frac{\gamma_{21} \vartheta_{0}}{2}\left(\int_{0}^{t} y_{F}(\varsigma) v(\varsigma) d \varsigma\right)^{2}+ \\
+\chi_{*} \int_{0}^{t} y_{F}(\varsigma) v(\varsigma) d \varsigma \pm \frac{\chi_{*}^{2}}{2 \gamma_{22} \vartheta_{0}} \geq \\
\geq-\frac{\chi_{*}^{2}}{2 \gamma_{22} \vartheta_{0}}=\eta_{02}^{2}, \eta_{02}=\operatorname{const}, \forall t>0 .
\end{gathered}
$$

Let us rewrite the integral from (21) like

$$
\begin{aligned}
& \int_{0}^{t}[S(u(\varsigma))-u(\varsigma)] v(\varsigma) d \varsigma= \\
= & \int_{0}^{t_{*}}[S(u(\varsigma))-u(\varsigma)] v(\varsigma) d \varsigma+ \\
+ & \int_{t_{*}}^{t}[S(u(\varsigma))-u(\varsigma)] v(\varsigma) d \varsigma,
\end{aligned}
$$

where $t_{*}$ is a time since which condition $|u(t)| \leq S_{0}$ is always fair. In this case for the first summand from (27) due to the boundednes of integrable function on a finite time interval we can write the following inequality

$$
\begin{gathered}
\left|\int_{0}^{t_{*}}[S(u(\varsigma))-u(\varsigma)] v(\varsigma) d \varsigma\right| \leq \eta_{03}^{2}, \\
\eta_{03}=\text { const }, \forall t \in\left[0 ; t_{*}\right] .
\end{gathered}
$$

Since at $t>t_{*}$ the identity $S(u(t))=u(t)$ will always hold for the second term from (27) following relation will be fair:

$$
\int_{t_{*}}^{t}[S(u(\varsigma))-u(\varsigma)] v(\varsigma) d \varsigma=0, \forall t \geq t_{*} .
$$

Thus, the obtained estimates (24), (26), (28) and (29) will provide following fair esimate for intgral inequality (19):

$$
\begin{gathered}
\eta(0, t) \geq-\sum_{i=1}^{3} \eta_{0 i}^{2}=-\eta_{0}^{2}, \\
\eta_{0}=\text { const }, \forall t>0 .
\end{gathered}
$$

The obtained estimate (30) ensures the fulfillment of positivity of the nonlinear non-stationary part of the equivalent system (17).

As a result, we have the nonlinear repetitive control system (1) - (10), (12), (16) in which with the help of the robust-periodic control law

$$
\begin{gathered}
u(t)=\left(u_{1}(t-T)+\gamma_{1} v(t) \tilde{\vartheta}(t)\right)+ \\
+\left(\gamma_{21} \int_{0}^{t} y_{F}(\varsigma) v(\varsigma) \tilde{\vartheta}(\varsigma) d \varsigma+\right. \\
\left.+\gamma_{22} y_{F}(t) v(t) \tilde{\vartheta}(t)\right) y_{F}(t), \\
\tilde{\tau} \frac{d \tilde{\vartheta}(t)}{d t}+\tilde{\vartheta}(t)=\vartheta(t), \tilde{\vartheta}(0)=0, \\
\vartheta(t)= \begin{cases}1, & \forall[S(u(t))-u(t)] v(t) \geq 0, \\
\vartheta_{0}, & \forall[S(u(t))-u(t)] v(t)<0,\end{cases}
\end{gathered}
$$

the fulfillment of the additional (12) and, as a consequence, the main (11) control goals are insurred.

Remark. The fast-acting correction filters (6) and (12) in the obtained control system are used. For the purpose to weaken the influence of peaks on the formation of control signals, similar to [Khalil, 2002], we limit the output of $O C F$ (6) with the help of nonlinearities of the saturation type. Then we can rewrite the technically feasible control law (31) as following:

$$
\begin{gathered}
u(t)=\left(u_{1}(t-T)+\gamma_{1} v(t) \tilde{\vartheta}(t)\right)+ \\
+\left(\gamma_{21} \int_{0}^{t} \operatorname{sat}\left(y_{F}\right)(\varsigma) v(\varsigma) \tilde{\vartheta}(\varsigma) d \varsigma+\right. \\
\left.+\gamma_{22} s a t\left(y_{F}(t)\right) v(t) \tilde{\vartheta}(t)\right) y_{F}(t), \\
\tau \tilde{\tau} \frac{d \tilde{\vartheta}(t)}{d t}+\tilde{\vartheta}(t)=\vartheta(t), \tilde{\vartheta}(0)=0, \\
\vartheta(t)= \begin{cases}1, & \forall[S(u(t))-u(t)] v(t) \geq 0, \\
\vartheta_{0}, & \forall[S(u(t))-u(t)] v(t)<0 .\end{cases}
\end{gathered}
$$

\section{Control System Simulation}

We consider the problem of control for lathe mechatronic feed module to illustrate the functioning quality of the obtained system (1) - (10), (12), (16), (32).

The initial data is as follows:

1. Setting action that determines the required processing profile of the product:

$$
\begin{gathered}
r(t)=r(t+T)=1.1- \\
=-0.1 \exp (-t)-|\cos (0.04 \pi t)|, \mu m .
\end{gathered}
$$




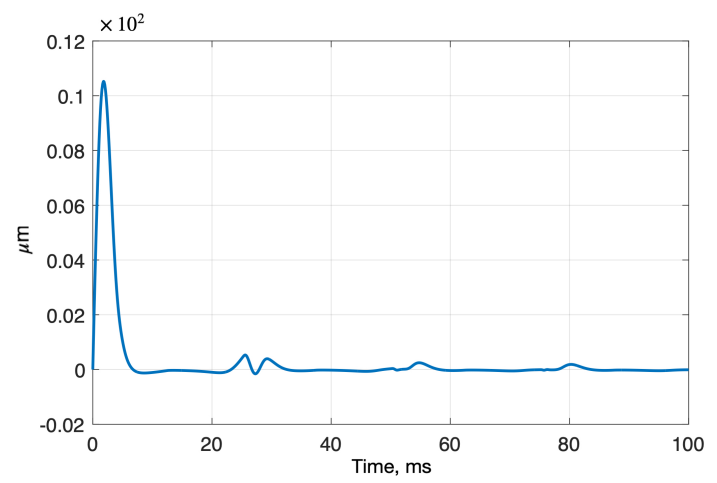

Figure 4. Tracking error

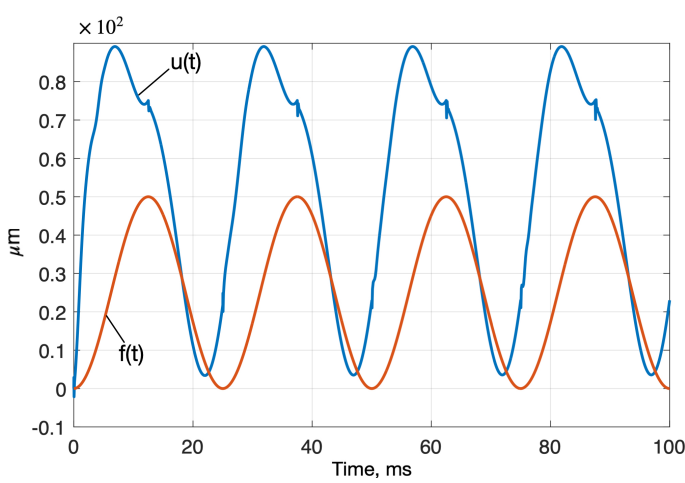

Figure 5. Control signal $\mathrm{u}(\mathrm{t})$ and the force disturbances $\mathrm{f}(\mathrm{t})$

2. Periodic force disturbance:

$$
f(t)=f(t+T)=0.5 \sin ^{2}(0.04 \pi t), \mu m .
$$

3. Numerical parameters of the control plant (1) - (4) corresponding to given class of uncertainty:

$$
\begin{gathered}
K=19, a_{1}=21.5, a_{2}=48, a_{3}=46.9 \\
a_{4}=10.2, S_{0}=5 .
\end{gathered}
$$

4. The transfer functions of $C C F$ and $O C F$ :

$$
W_{F}(p)=\left(\frac{0.17 p+1}{0.01 p+1}\right)^{3} .
$$

In the course of computational experiments we obtained the results are depicted in Fig. 3-5. The parameters of the control loop (32) in order to increase the speed of the system were selected with the following values:

$$
\begin{aligned}
& \gamma_{1}=40, \gamma_{21}=200, \gamma_{22}=150, T=25, \\
& \vartheta_{0}=0.5, \tilde{\tau}=15, \\
& \text { sat }\left(y_{F}(t)\right)= \begin{cases}5, & y_{F}(t)>5 \\
y_{F}(t), & \left|y_{F}(t)\right| \leq 5 \\
-5, & y_{F}(t)<-5 .\end{cases}
\end{aligned}
$$

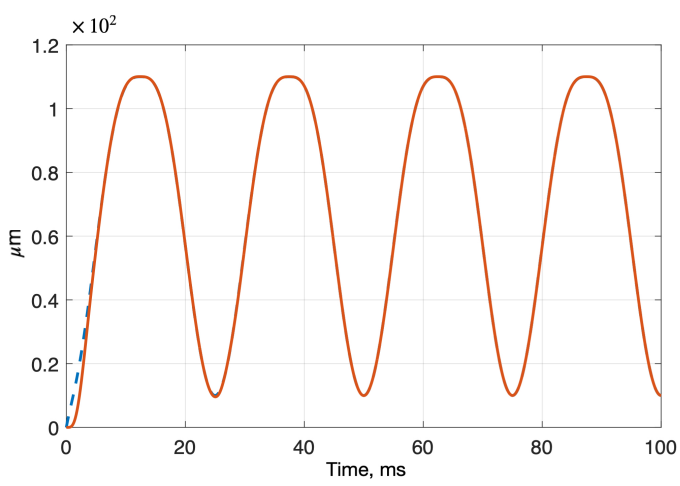

Figure 3. Required (dotted lines) and actual (solid lines) displacements of the executive body in the control system (1) - (10), (12), (16), (32) - (37)

The obtained dynamic characteristics indicate a good quality of the proposed control system. With the selected coefficients of the nonlinear repetitive regulator (32), (37), the parameters (35) of the controlled plant (1) and external cyclic noises (34), the system provides high-precision tracking of the required command signal (33), which means the fulfillment of the control goal (11).

The graphs of the system tracking errors with the plant parameters corresponding the upper bound of parametric uncertainty (2)

$$
\begin{gathered}
K=K^{+}=19.5, a_{1}=a_{1}^{+}=23.5, \\
a_{2}=a_{2}^{+}=64, a_{3}=a_{3}^{+}=50, \\
a_{4}=a_{4}^{+}=16.5
\end{gathered}
$$

are shown in Fig. 6,a. Fig. 6,b shows tracking error when the plant parameters are equal to the lower bound of uncertainty (2):

$$
\begin{gathered}
K=K^{+}=18.56, a_{1}=a_{1}^{+}=23.5, \\
a_{2}=a_{2}^{+}=41.4, a_{3}=a_{3}^{+}=45.7, \\
a_{4}=a_{4}^{+}=8.5 .
\end{gathered}
$$

It should be noted that the value of the control error when changing parameters of the control plant from the initial moment of time does not exceed $3.5 \mu \mathrm{m}$ (3\%). In the steady state (6 seconds after the systems start operating), the tracking error in all cases does not exceed $0.5 \mu m(1 \%)$. 


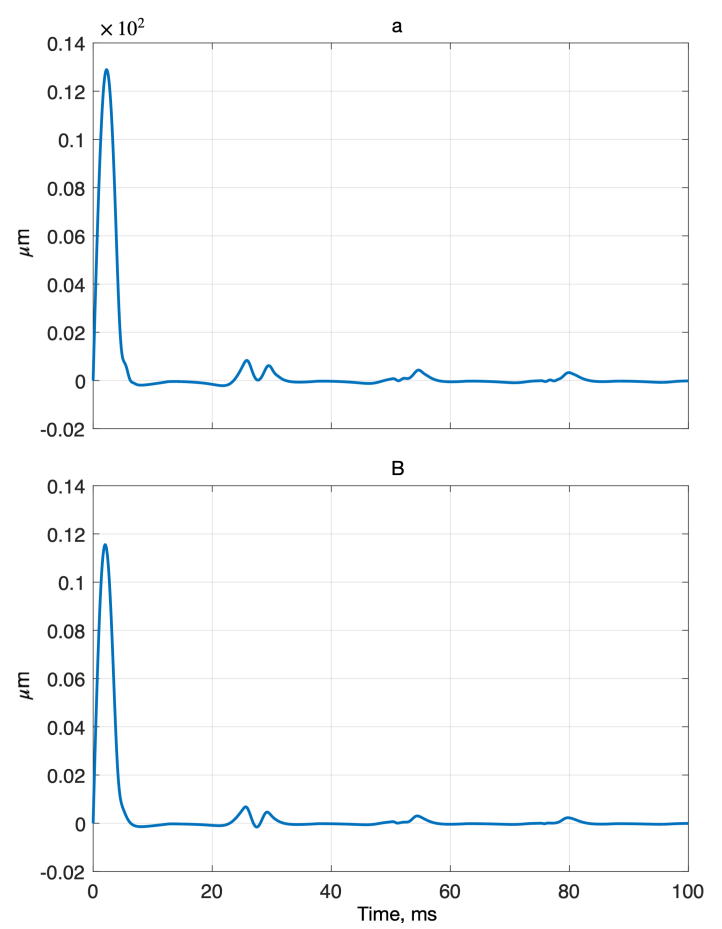

Figure 6. Tracking errors at different parameters of the control plant

\section{Conclusion}

The nonlinear-periodic controller has been developed on the basis of V.M. Popov's hyperstability criterion and L-dissipativity conditions for the control system of the mechatronic module of lathes.

The synthesized nonlinear repetitive control loop ensures invariance of the system to changes in parameters of controlled plant (when they change within the uncertainty class), as well as to a significant increase in external force disturbances.

\section{Acknowledgements}

The reported study was funded by RFBR (project No. 20-08-00712).

\section{References}

Alsubaie, M., Alhajri, M., and Altowaim, T. (2018). Repetitive Control Uncertainty Conditions in State Feedback Solution. Intelligent Control and Automation, 9, pp. 95-106.

Eremin, E. L., Nikiforova, L. V., and Shelenok, E. A. (2020). Combined System Controller with an Implicit Reference for Output Control of a Srtructurally Indeterminate Non-Affine Plant with an Unknown State Delay. Information Science and Control Systems, 4(66), pp. 118-128.

Eremin, E. L., and Shelenok, E. A. (2018). Nonlinear-Periodic Control System for Non-Affine Multi-Connected Plant with State Delay. Cybernetics and Physics, 7:4, pp. 195-203.

Eremin, E. L., and Shelenok, E. A. (2018). NonlinearPeriodic Control System for Non-Affine Stationary
Plant with Statement Delay. In Proc. International Multi-Conference on Industrial Engineering and Modern Technologies (FarEastCon), Vladivostok, Russia, Oct. 3-4, pp. 1-7.

Eremin, E. L., and Shelenok, E. A. (2018). NonlinearPeriodic System for Non-Affine in Control Stationary Plant. Information Science and Control Systems, 1(55), pp. 130-141.

Ghosh, J., and Paden, B. (2000). Nonlinear repetitive control. IEEE Transactions on Automatic Control, 45:5, pp. 949-954.

Hara, S., Yamamoto, Y., Omata, T., and Nakano, M. (1988). Repetitive Control System: A New Type Servo System for Periodic Exogenous Signals. IEEE Transactions on Automatic Control, 33:7, pp. 659-668.

Ishii, H., and Yamamoto, Y. (1998). Periodic Compensation for Sampled-Data N"O Repetitive Control. In Proc. 37th IEEE Conference on Decision \& Control, Tampa, Florida, USA, Oct. 18, pp. 331-336.

Jiang, Y. A., Clements, D. J., and Hesketh, T. (1995). Adaptive Repetitive Control of Nonlinear Systems. In Proc. 34th IEEE Conference on Decision \& Control, New Orleans, LA, USA, Dec. 13-15, pp. 1708-1713.

Khalil H. K. (2002). Nonlinear Systems. Prentice Hall, New Jersey.

Li, C. J., and Li, C. Y. (1992). On-Line Roundness Error Compensation via P-Integrator Learning Control. Journal of Engineering for Industry, (114), pp. 476480.

Ma, G., Liu, X., Pagilla, P. R., and Ge, S. S. (2020). Asynchronous repetitive control of switched systems via periodic event-based dynamic output feedback. IMA Journal of Mathematical Control and Information, 37:2, pp. 644-673.

Nikolova, N. G. (2019). Examination of a repetitive process control system. IOP Conference Series: Material Science and Engineering, (618), pp. 012-013.

Nikolsky, A. A. (2016). Accurate self-learning electric drives for non-circular turning machines. Advanced solutions, Moscow.

Nikolsky, A. A. (2012). High-precision multi-circuit self-learning mechatronic systems with piezo compensators for non-circular turning machines. Elektrichestvo, (7), pp. 52-57.

Ramos, G. A., Ruget, R. I., and Costa-Castelló R. (2020). Robust Repetitive Control of Power Inverters for Standalone Operation in DG Systems. IEEE Transactions on Energy Conversion, 35:1, pp. 237-247.

Toujeni, N., Chaouki, M., and Gasmi, M. (2020). Repetitive Control based on Integral Sliding Mode Control of Matched Uncertain Systems. International Journal of Advanced Computer Science and Applications, 9:12, pp. 446-455.

Yao, W. S., Tsai, M. C., and Yamamoto Y. (2013). Implementation of Repetitive Controller for Rejection of Position-Based Periodic Disturbances. Control Engineering Practice, 21:9, pp. 1226-1237. 
Yu, P., Wu, M., She, J., and Lei, Q. (2016). Robust Repetitive Control and Disturbance Rejection Based on Two-Dimensional Model and EquivalentInput-Disturbance Approach. Asian Journal of Con- trol, 18:6, pp. 1-11.

Yurkevich, V. V. (2001). The system for predicting the accuracy of lathes. Vestnik mashinostroeniya, (8), pp. 44-48. 